\title{
Carbon black directed synthesis of ultrahigh mesoporous carbon aerogels
}

Carlos Macías ${ }^{1}$, Marta Haro ${ }^{2}$, José B. Parra ${ }^{2}$, Gloria Rasines ${ }^{1}$, Conchi O. Ania ${ }^{2} *$

${ }^{1}$ Nanoquimia S.L., P.L La Minilla, La Rambla, 14540 Córdoba (Spain)

${ }^{2}$ Instituto Nacional del Carbón, INCAR-CSIC, Apdo. 73, 33080 Oviedo, Spain

\begin{abstract}
A simple modification of the conventional sol-gel polymerization of resorcinol-formaldehyde mixtures allowed a facile preparation of ultrahigh mesoporous carbon gels. In the conventional synthesis the growth of the cluster polymer particles leading to the development of the porosity is controlled by the $\mathrm{R} / \mathrm{C}$ ratio. In the presence of a carbon conductive additive, the polymerization of the reactants proceeded through the formation of less-branched polymer clusters resulting in carbon gels with large pore volumes within the micro/mesoporous range. The obtained materials displayed unusual heterogeneous pore systems characterized by large mesopores interconnected by necks of variable sizes, along with an enhanced electrical conductivity provided by the carbon black additive. The gels showed stable electrochemical response in neutral aqueous electrolyte, being reversibly charged/discharged at large potential windows, without significant losses in the current density, chemical modifications or structural collapse. The enhanced life cycle of these electrodes makes them good candidates for their use in electrochemical applications where a fast response and high cycleability is required.
\end{abstract}

*Corresponding author. Tel/Fax: +34 985 118846/ +34 985 297662. E-mail address: conchi.ania@incar.csic.es (C.O. Ania) 


\section{Introduction}

Modern carbons are emerging as promising candidates in many science and engineering fields, particularly in the energy storage and environmental remediation arena, as a result of an unforeseen control in their physicochemical and structural properties during the synthesis and processing [1-3]. Among them, carbon gels (xerogel, aerogel and cryogels) appear to be within the most suitable materials in these fields due to their relatively low cost and unique combination of adequate properties that may be tuned during the synthesis and processing [49]. Indeed, since the first studies of Pekala and coworkers [10] numerous articles have appeared in the literature for the last two decades or so, describing various synthesis and processing conditions and their effects on the final structure (morphology, texture, composition) of the obtained materials and consequently on their performance [4-10]. A detailed review on this matter has been published by Ritter and co-workers in 2003 [4]. Most of these works have reported the unique physical, chemical and electrochemical properties of these lightweight and high performance engineering materials, showing potential applications in sectors such as: adsorbents for gas separation [11], catalyst supports [12,13], energy storage devices[14-17], capacitive deionization for water purification [18-20], etc. Carbon gels are mostly synthesized following the catalyzed polycondensation of resorcinolformaldehyde mixtures in water, although some other alternative routes have been described using harmless and naturally available compounds as precursors [21, 22].

Despite carbon gels typically exhibit electrical conductivities higher than other types of aerogels (which are generally insulating materials) and most porous carbons [23, 24] (excluding carbon nanotubes, graphenes or graphitic carbons), the conductivity of resorcinolformaldehyde derived carbon gels is still rather limited. Thus, for most electrochemical applications, the use of carbon back as conductive additive (CCA) is still recommended (or 
needed) in the manufacturing of the electrodes so as to limit the resistance of the system due to the conductivity limitation of the electrode materials [23-26].

The objective of this work was to synthesize carbon aerogels with controlled porosity and high electrical conductivity and to explore their potential use in electrochemical applications. The sol-gel polymerization of the gel precursors was carried out in the presence of a carbon conductive additive (i.e., carbon black); this allowed the fabrication of carbon gels with extremely high pore volumes within the micro/mesopore range, largely exceeding the porous features commonly reported for carbon gels. Although the benefits of the use of conductive carbon blacks in the manufacturing of carbon electrodes have been reported in the literature $[25,26]$, herein obtained results have shown that incorporating the CCA before the sol-gel reaction promotes outstanding changes in the texture of the final carbon/carbon nanocomposites, beyond the expected improved electrical conductivity.

\section{Experimental}

\subsection{Materials Synthesis}

The carbon gels were synthesized by the sol gel polymerization of resorcinol (R) and formaldehyde $(\mathrm{F})$ in water $(\mathrm{W})$, using sodium carbonate $(\mathrm{C})$ as catalyst, carbon black $(\mathrm{CB}$, Superior Graphite Co.) as conductive additive, and following an experimental procedure inspired from the method described by Pekala and co-workers [10]. Details of the conventional synthesis have been reported elsewhere [27]. Briefly, the precursors were mixed in sealed glass moulds under magnetic stirring and allowed to undergo gelation and aging in an oven at $40^{\circ} \mathrm{C}$ for $24 \mathrm{~h}$ and $70{ }^{\circ} \mathrm{C}$ for $120 \mathrm{~h}$. Subsequently a controlled water-acetone exchange was carried out, and then the pieces were dried under $\mathrm{CO}_{2}$ supercritical conditions. Finally, the aerogels were pyrolyzed under nitrogen atmosphere at $800{ }^{\circ} \mathrm{C}$ (unless otherwise indicated). The resorcinol/catalyst (R/C) ratio was varied (i.e., 25, 50, 100 and 200) in order 
to produce samples with different porous texture, while the molar ratio $\mathrm{R} / \mathrm{F}$ was fixed at 0.5 , the $\mathrm{R} / \mathrm{W} 0.06$ and the $\mathrm{CB} /(\mathrm{R}+\mathrm{F})$ was 5 and $10 \mathrm{wt} . \%$ (values were taken as representative of commonly used percolator mass ratio in electrochemical applications). The nomenclature of the samples was Gx-CBy, being $x$ the corresponding R/C value (i.e., G25-CB) and $y$ the $\mathrm{CB} /(\mathrm{R}+\mathrm{F})$ ratio (i.e., G25-CB10). To evaluate the thermal stabilization of the organic gels upon pyrolysis, selected gel/CCA composites were also treated at 500 and $1000{ }^{\circ} \mathrm{C}$, obtaining series Gx-CByT, where T stands for the pyrolysis temperature. For the sake of comparison, a second series of carbon gels was prepared by the same synthetic route without the incorporation of the carbon black (series Gx).

\subsection{Electrochemical characteristics}

Electrodes were prepared by mixing the carbon material $(90 \mathrm{wt} . \%)$ with a binder $(10 \mathrm{wt} . \%$, polyvinylidene fluoride). These mixtures were pressed into $10-15 \mathrm{mg}$ pellets. Three-electrode cells were assembled on a Teflon Swagelok system using a carbon pellet as working electrode, a high surface area activated carbon pellet (Maxsorb, $\mathrm{S}_{\mathrm{BET}}=2600 \mathrm{~m}^{2} \mathrm{~g}^{-1}$ ) as counter electrode and saturated mercury/mercurous sulfate reference electrode. All potential values are expressed vs. the normal hydrogen electrode (NHE). Aqueous $0.5 \mathrm{~mol} \mathrm{~L}^{-1} \mathrm{Na}_{2} \mathrm{SO}_{4}$ was used as electrolyte. The carbon pellets were dried at $60^{\circ} \mathrm{C}$ under vacuum overnight and vacuum-impregnated before assembling the cells. Several electrochemical measurements were performed on each carbon aerogel electrode, using a computer controlled potentiostat/galvanostat (Biologic VMP-3). Aging experiments were conducted on symmetric cells -capacitors- in aqueous electrolyte- Initially the cells were galvanostatically charged/discharged between $0-1.4 \mathrm{~V}$ and at a specific current density of $500 \mathrm{~mA} \mathrm{~g}^{-1}$ over six cycles for evaluating the specific cell capacitance. Afterwards, the cells were charged up to 1.4 $\mathrm{V}$ followed by holding the voltage for $12 \mathrm{~h}$. The cell was then cycled again and the 
procedure was repeated until an overall holding period of $500 \mathrm{~h}$ was reached. The resistivity measurements of powdered and pressed carbons were carried out at atmospheric pressure, using a home-made apparatus. Measurements were based on the van der Pauw technique [28], which involves the application of a bias current of $50 \mathrm{~mA}$ to the carbon pellets and measuring the voltage drop, using a four probe configuration.

\subsection{Nanotextural and Chemical Characterization}

The nanotexture of the prepared gels was characterized by measuring the $\mathrm{N}_{2}$ adsorption isotherms at $-196^{\circ} \mathrm{C}$ (ASAP 2010, Micromeritics). Before the experiments, the samples were outgassed under vacuum (ca. $10^{-3}$ torr) at $120{ }^{\circ} \mathrm{C}$ overnight. The isotherms were used to calculate the specific surface area, $\mathrm{S}_{\mathrm{BET}}$, total pore volume, $\mathrm{V}_{\mathrm{T}}$, and pore volumes using the DR formulism and non-local density functional theory (DFT). SEM/EDX analyses were performed with a FE-SEM apparatus (QuantaSEM, FEI). Elemental analysis was carried out in LECO CHNS-932 and LECO VTF-900 automatic analyzers.

\section{Results and discussion}

\subsection{Synthesis of the aerogel/CCA materials}

The sol-gel procedure used in this work was a simple modification of the conventional procedure reported by Pekala and co-workers [10], consisting on carrying out the sol-gel polymerization of the precursors (resorcinol and formaldehyde) in the presence of a carbon conductive additive on the mixture. As conductive additive, a carbon black (Superior Graphite, Co.) commonly used in electrochemical applications was selected; this material is characterized by low ash, moisture, sulfur and volatiles contents, as well as a high electrical conductivity provided by its ordered structure (see TEM images in Fig. 1). According to literature, the polymerization mechanism of resorcinol-formaldehyde (RF) gels is typically 
controlled by the addition reaction between the precursors to form hydroxylmethyl derivatives, followed by condensation to form methylene ether bridged compounds, and cluster growth [4-7]. The initial cluster formation is followed by the spinodal decomposition of the growing clusters that promptly become unstable and thus gelation occurs. For our systems, gelation occurred regardless the amount of CCA added (i.e. 5 or $10 \mathrm{wt} \%$ ), indicating that the cross-linking of the clusters (that leads to the stiffing of the gel) progressed adequately upon incorporation of the CCA. It should be mentioned that the loading of the CCA was selected considering commonly percolator mass ratio reported in the literature in electrochemical applications [23-26]. Above $10 \mathrm{wt} . \%$, the dispersion of the carbon black in the reactants mixture was not uniform, which impeded the homogeneous sol-gel reaction. At lower loadings (ca. 0.5-2wt.\%) the effect on the electrical conductivity of the aerogel electrodes was almost negligible, thus these samples were no longer investigated. The lack of swelling during the solvent exchange stage also indicated that cross-linking reactions were sufficiently completed in the presence of the CCA particles. The appearance of the aerogels changed with the synthesis variables; before pyrolysis, conventional RF aerogels were opaque and dark for low $\mathrm{R} / \mathrm{C}$ ratios (i.e. series 25 and 50) turning translucent light red for the higher R/C ratio (Figure S1. in Supplementary File). After pyrolysis, all the materials become opaque and dark, regardless the synthesis conditions. On the other hand, all the aerogels prepared in the presence of the carbon black additive were black and opaque regardless the $\mathrm{R} / \mathrm{C}$ ratio and before the pyrolysis treatment.

Scanning microscopy images also showed that the incorporation of the carbon black did not modify the gels structure compared to conventional synthesis (Fig. 2). Moreover, TEM images in Fig. 1 showed that the gel/CCA composite exhibit domains of graphitic order provided by the carbon black arranged within the amorphous carbon matrix characteristic of the materials prepared by the conventional synthesis. 


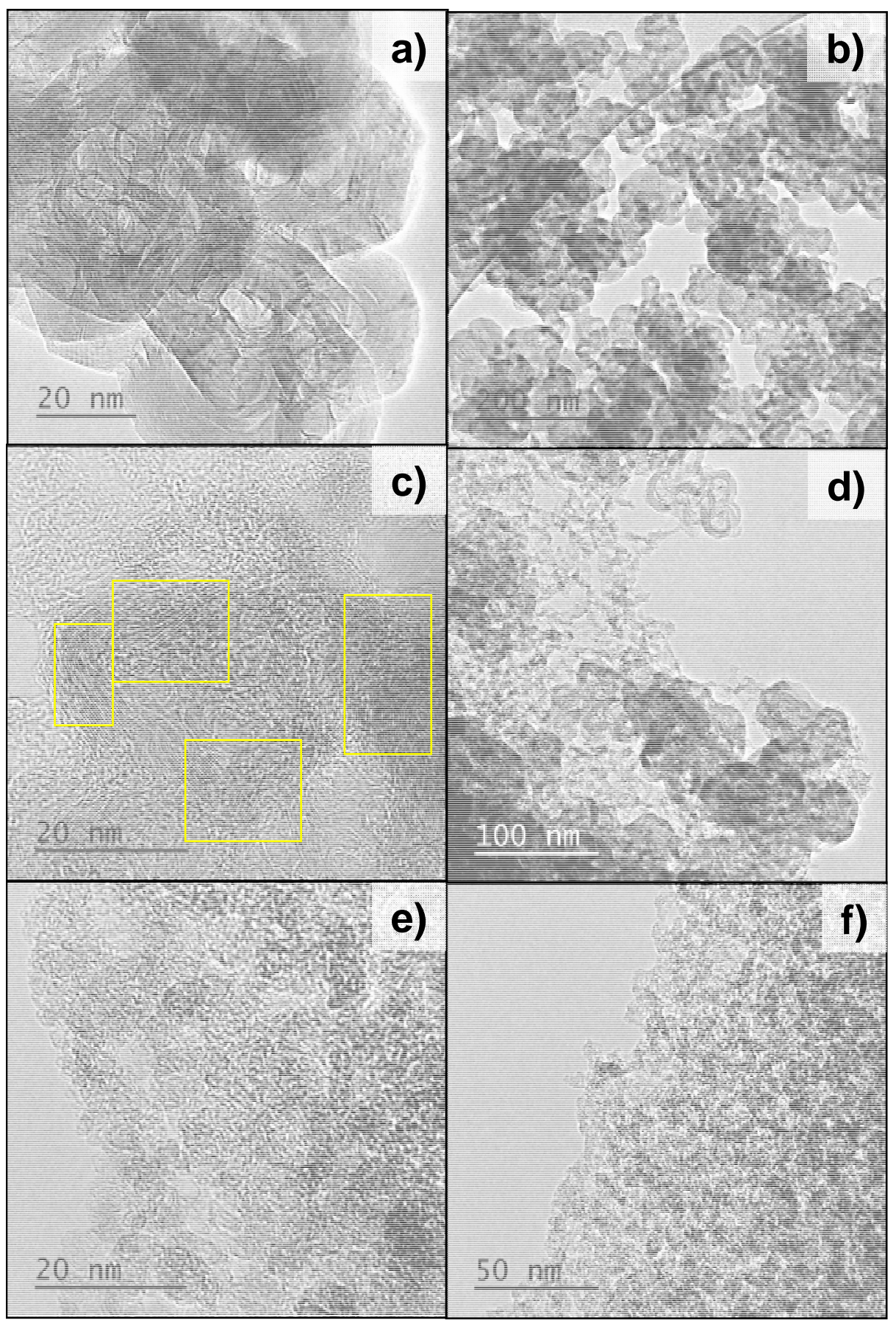

Fig. 1. Transmission electron micrographs of the carbon conductive additive $(a, b)$ and the carbon aerogels prepared in the presence $(\mathrm{c}, \mathrm{d}$, representative images corresponding to G200-CB10) and absence (e,f, representative images corresponding to G200) of the carbon black. For clarity, areas showing the structural graphitic order in the gel/CCA sample are indicated by the squares. 


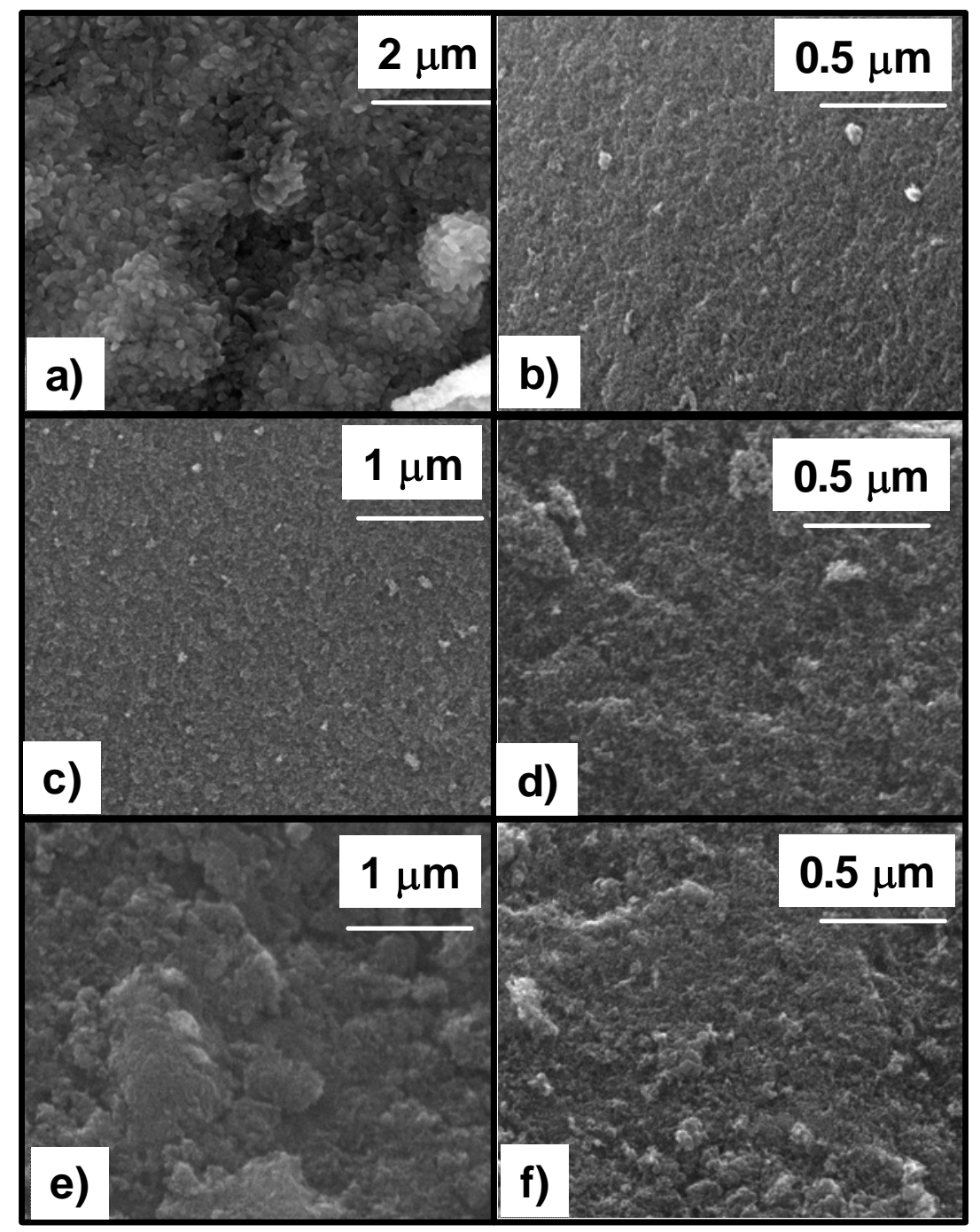

Fig. 2. Scanning electron micrographs of selected carbon aerogels: a) G25; b) G50; c, d) G200; e, f) G200-CB10.

Thermal annealing of the dried aerogels under inert atmosphere at 500, 800 and $1000{ }^{\circ} \mathrm{C}$ preserved the monolithic shape of the gels, although some cracks appeared at the surface (Figure S1). This was particularly evident for the samples prepared from the highest R/C ratio, suggesting a rather uniform shrinkage for all the samples. After pyrolysis, all the gels were bright black and showed rather good mechanical strength. Moreover, the CCA appeared 
to enhance the mechanical properties of the materials prepared from $\mathrm{R} / \mathrm{C}$ above 100 , otherwise brittle and difficult to handle without damage.

The evolution of the porous features of the synthesized materials was very dependent on the incorporation of the CCA before the sol-gel reaction. Fig. 3 and Table 1 show the main textural parameters determined by nitrogen adsorption at $-196^{\circ} \mathrm{C}$.
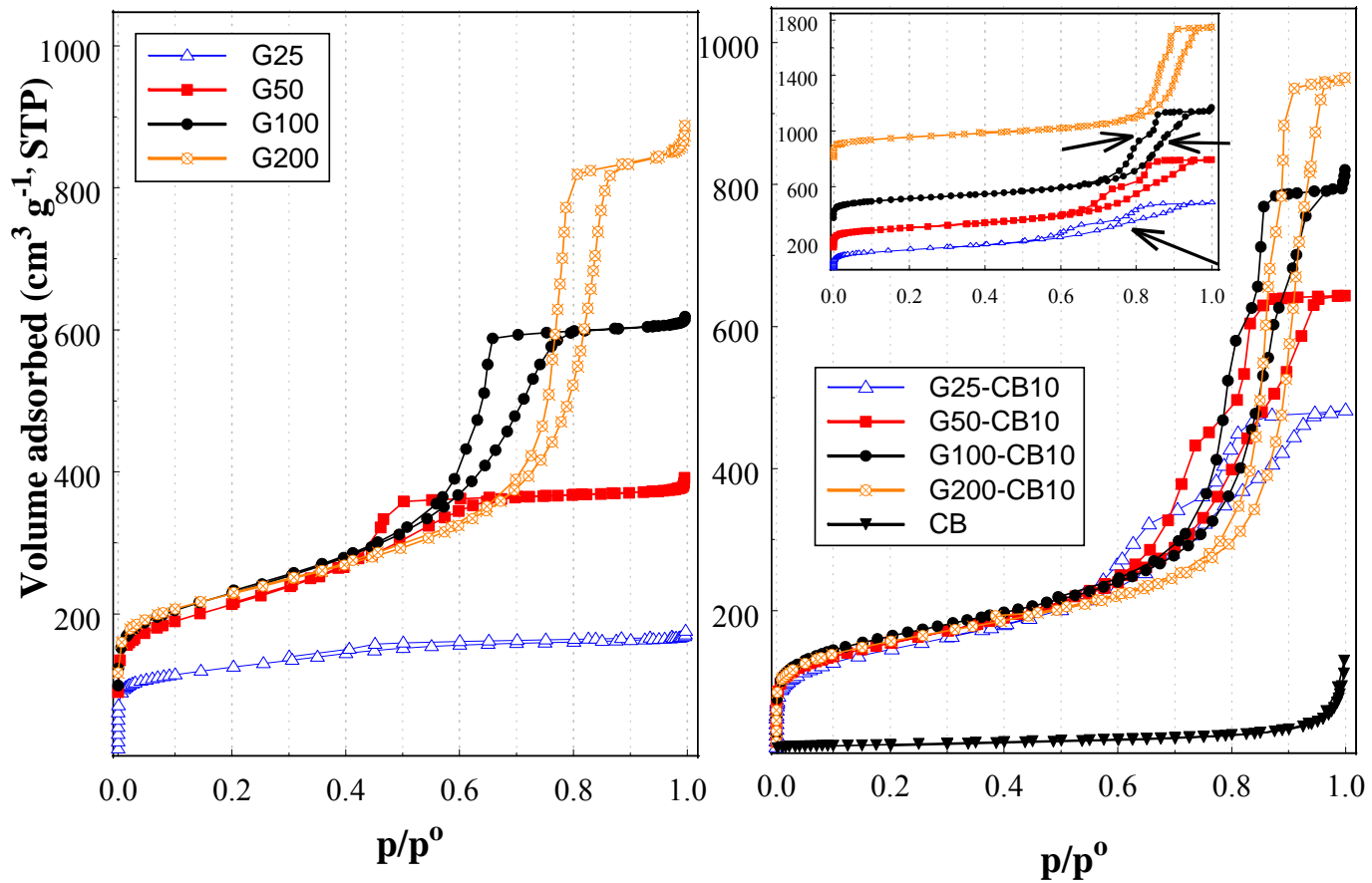

Fig. 3. Nitrogen adsorption isotherms at $-196^{\circ} \mathrm{C}$ of the series of carbon gels prepared with (right) and without (left) the incorporation of the carbon conductive additive. Inset: isotherms have been shifted for clarity.

For the carbon gels synthesized following the conventional procedure, the expected influence of the $\mathrm{R} / \mathrm{C}$ ratio in the porous network connectivity and the textural parameters (micro/mesopore balance, surface area and average pore width) was observed [4-9] Briefly, a gradual shift from type I (predominant microporosity + narrow mesopores) towards type IV adsorption isotherms (low micro/mesopore ratio) was observed with the $\mathrm{R} / \mathrm{C}$ ratio, accompanied by an increase in the specific surface area and pore volumes. This indicates that 
the development of a mesoporous structure is favored when the reactivity of the carbon precursors is lowered (reducing the amount of catalyst). Prominent hysteresis loops appearing at high relative pressures and a non-negligible microporosity contribution were also observed, which according to literature are characteristics of the so-called colloidal gels prepared using high $\mathrm{R} / \mathrm{C}$ ratio $[5,6]$.

Table 1. Porosity parameters obtained from the $\mathrm{N}_{2}$ adsorption isotherms at $-196{ }^{\circ} \mathrm{C}$ for the gels prepared with and without the incorporation of the carbon conductive additive.

\begin{tabular}{|c|c|c|c|c|c|}
\hline & $\mathrm{S}_{\mathrm{BET}}$ & $\mathrm{V}_{\text {TOTAL PORES }}$ & Wo (DR) & $\mathrm{V}_{\text {MESO }}$ & Mean mesopore \\
\hline & {$\left[\mathrm{m}^{2} \mathrm{~g}^{-1}\right]$} & {$\left[\mathrm{cm}^{3} \mathrm{~g}^{-1}\right]$} & {$\left[\mathrm{cm}^{3} \mathrm{~g}^{-1}\right]$} & {$\left[\mathrm{cm}^{3} \mathrm{~g}^{-1}\right]$} & size $[\mathrm{nm}]$ \\
\hline Carbon Black & 41 & 0.13 & 0.02 & 0.06 & -- \\
\hline G25 & 455 & 0.26 & 0.17 & 0.09 & 4 \\
\hline G50 & 760 & 0.59 & 0.35 & 0.24 & 5 \\
\hline G100 & 820 & 0.95 & 0.29 & 0.66 & 8 \\
\hline G200 & 830 & 1.33 & 0.31 & 1.02 & 12 \\
\hline G25-CB10 & 535 & 0.74 & 0.18 & 0.56 & $7-13$ \\
\hline G50-CB10 & 542 & 1.09 & 0.20 & 0.89 & $9-15$ \\
\hline G100-CB10 & 592 & 1.22 & 0.20 & 1.02 & $13-17$ \\
\hline G200-CB10 & 559 & 1.46 & 0.20 & 1.26 & $17-22$ \\
\hline G50-CB5 & 633 & 0.88 & 0.21 & 0.67 & $5-8$ \\
\hline G50-CB10-T500 & 569 & 1.02 & 0.21 & 0.81 & $9-15$ \\
\hline G50-CB10-T1000 & 498 & 1.10 & 0.20 & 0.90 & $12-18$ \\
\hline
\end{tabular}


The incorporation of the CCA allowed the preparation of ultrahigh mesoporous carbon gels as seen by the nitrogen adsorption isotherms (Fig. 3). All the samples exhibited type IV isotherms regardless the $\mathrm{R} / \mathrm{C}$ ratio, along with large total pore and mesopore volumes (Table 1). It is interesting to remark that the nitrogen adsorption isotherms of the Gx-CBy composites displayed a singular shape characterized by a curvature both in the adsorption and desorption branches (marked by arrows in Fig. 3 and 4). The onset of the curvature moved upwards to higher pressures with the $\mathrm{R} / \mathrm{C}$ ratio, ranging from 0.75 for $\mathrm{G} 25-\mathrm{CB} 10$ to 0.88 for G200-CB10.

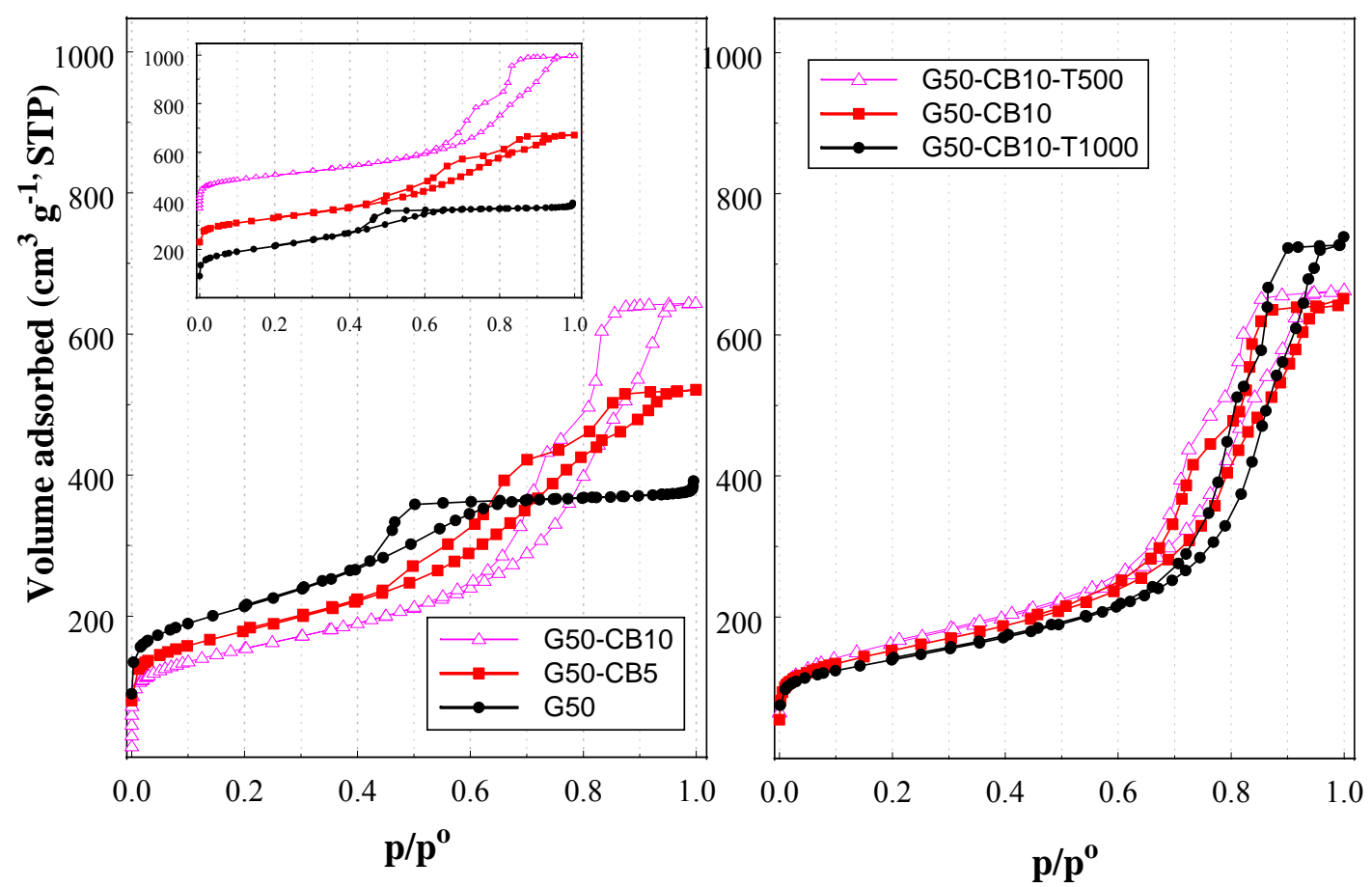

Fig. 4. Nitrogen adsorption isotherms at $-196^{\circ} \mathrm{C}$ of the series of carbon gels prepared with different amounts of CCA (left), and effect of the pyrolysis temperature on the prepared gel/CCA materials (right) Inset. Isotherms have been shifted for clarity.

Such two-step adsorption/desorption suggests that these materials have a porous structure with both open and blocked cylindrical mesopores [29-31] where the main cavities (mesopores) are interconnected through narrower necks. The double step condensation in the adsorption branch would correspond to the consecutive condensation in pore necks and 
cavities, whereas the curvature of the desorption branch would account for emptying of the neck and the cavity [32,33].

In the case of samples G25-CB10 and G50-CB10 (low $\mathrm{R} / \mathrm{C}$ ratio), the adsorption and desorption branches of the $\mathrm{N}_{2}$ isotherms are very sloping. Both show a well-defined curvature (inflection point) with large hysteresis loops -starting from relative pressures of 0.4 and 0.7 , respectively, up to 1-. These features are in agreement with pore systems characterized by heterogeneous pore distributions where mesopores having different width bodies are interconnected through a wide range of openings of narrower sizes [29-31]. For higher R/C ratio (samples G100-CB10 and G200-CB10), the adsorption and desorption branches are more vertical and almost parallel over the gas uptake, indicating a more homogenous distribution of pore sizes compared to G25-CB10 and G50-CB10. The curvature of the adsorption/desorption branches and the position of the hysteresis loops suggest that the pore network of these materials is composed of cylindrical mesopores with uniform bodies, connected by narrower cylindrical necks of uniform widths.

As for the textural parameters, pore volumes of the gel/CCA composites largely exceeded the values obtained with the conventional synthetic route (Table 1). Specific surface areas did not follow the same trend, with smaller values for the materials prepared in the presence of the CCA. Moreover, whereas surface areas discretely increase with the $\mathrm{R} / \mathrm{C}$ ratio for the control Gx series (only G200 break this trend), values varied within a short range for the materials prepared in the presence of the CCA.

This trend was rather expected for the control Gx materials, since specific surface area is closely related to the microporosity, which according to literature is controlled by the $\mathrm{R} / \mathrm{C}$ ratio in conventional synthesis $[5,6]$. Conversely, all the gel/CCA samples presented similar micropore volumes (Table 1) regardless the $\mathrm{R} / \mathrm{C}$ ratio. This suggests that the incorporation of the carbon black additive mainly affects the formation of large mesopores (voids between the 
particles), whereas the microporosity (voids inside the particles) remained rather the same for a given amount of CCA added.

Further information on the nanotexture of the synthesized gels was obtained from the analysis of the pore size distribution using the non-local density functional theory. For the series prepared by the conventional sol-gel method, the typical monodispersed distribution of pore sizes in the mesopore range was obtained, with the average mesopore size increasing with the $\mathrm{R} / \mathrm{C}$ ratio and ranging from 5 to $12 \mathrm{~nm}$ [27]. When the CCA was incorporated, a multimodal distribution was obtained regardless the $\mathrm{R} / \mathrm{C}$ ratio, with increasing average mesopore sizes between 7-22 $\mathrm{nm}$ (Table 1). This behavior is attributed to the role of the carbon black additive during the sol-gel synthesis, which would induce the formation of large polymeric particles that lead to mesopore channels interconnected by narrower necks. These features allow a finetuning control of the micro/mesoporosity of the gels modified with a conductive additive. To analyze the effect of the amount of carbon black incorporated during the synthesis on the porosity, two identical aerogels were prepared adding 5 and $10 \mathrm{wt} . \%$ of the CCA while keeping constant the rest of parameters (samples G50-CB5 and C50-CB10). The corresponding gas adsorption isotherms are shown in Fig. 3. Increasing the amount of carbon black led to higher total pore volumes and to an enlargement of the average mesopore size. This is evidenced by the shift of the hysteresis loop to higher condensation pressures in the adsorption isotherms. It may be inferred then that the pore width is not only controlled by the $\mathrm{R} / \mathrm{C}$ ratio (as in conventional synthesis) but also by the amount of carbon black.

For the sample prepared with the lowest amount of CCA (carbon G50-CB5), three distinctive regions can be clearly identified as fused hysteresis loops in the nitrogen adsorption isotherm (Fig. 3). This points out the occurrence of a large pore blocking effect in the network, likely due to mesopores interconnected by narrower necks of various sizes. When the amount of carbon black increased (sample G50-CB10), only two stages (fused hysteresis loops) are 
distinguished, along with an upward shift in the relative pressure of the loops in the adsorption isotherms. This reveals that the size of the pore necks is enlarged with the amount of CCA. Interestingly, both samples showed a similar curvature in the adsorption isotherm appearing at relative pressures of ca. 0.8 (see inset in Fig. 3). Consequently it seems that the size of the large mesopores is mainly controlled by the $\mathrm{R} / \mathrm{C}$ ratio (being similar in G50-CB5 and G50-CB10), whereas the CCA would control the size of the pore necks connecting the main cavities. Pore systems showing fused hysteresis loops have been reported for chemically modified microporous glasses $[29,30]$ and silica materials consisting of spherical mesopores connected through narrow openings [34].

Based on the porosity evolution of the gel/CCA composites, it can be inferred that the incorporation of the conductive additive strongly affects the polymerization mechanism of the precursors. In the conventional synthesis, highly reactive systems are favored for low $\mathrm{R} / \mathrm{C}$ molar ratio, where the rate of formaldehyde consumption is accelerated leading to dense branched clusters, which easily undergo spinodal decomposition. In the presence of the aggregates of CCA, the large pore volumes and hysteresis loop suggest that the polycondensation of the monomers is slowed down. This would suppress the formation of the hydroxymethly derivates that allow the cross-linking of the structure (in a similar way as the effect of the $\mathrm{R} / \mathrm{C}$ value in the conventional synthesis [4-6, 34], generating less branched clusters that are more stable in the nucleation regime, allowing the colloidal particles to aggregate and assemble together in larger and weakly branched clusters. As a result, the carbon yields (after pyrolysis) obtained for the gel/CCA composites were larger (ca. 59-63 \% for $\mathrm{Gx}-\mathrm{CBy}$ ) than those of the gels obtained in the conventional synthesis for all $\mathrm{R} / \mathrm{C}$ values (ca. $50 \%$ for series $\mathrm{Gx}$ ).

During the clusters formation and growth, the carbon black aggregates (of a few nm width) would act as an ionomer finely dispersed within the primary particles of the carbon precursor 
monomers, creating larger and poorly cross-linked polymer particles but providing additional electrical conductivity.

The high pore volumes of the gel/CCA samples were almost unaffected by the pyrolysis temperature (Fig. 3), unlike the trend reported for carbon gels prepared by the conventional sol-gel route [4-6]. In a typical synthesis, the pyrolysis temperature may cause a structural collapse due to the surface tensions arising during gas evolution after the decomposition of the organic gels components (i.e. $\mathrm{OH}$ moieties, $\mathrm{H}_{2} \mathrm{O}, \mathrm{CO}_{2}, \mathrm{CO}$ and other organic molecules). This effect is generally more remarkable in gels composed of small nodules (low R/C ratio) and narrow pores, which are less resistant to the surface tensions provoked during gas evolution. Elemental analysis confirmed the large loss of oxygen, hydrogen and other volatiles from the carbonaceous structure during pyrolysis, with carbon contents increasing from 50-75 wt.\% (as-prepared gels with increasing $\mathrm{R} / \mathrm{C}$ ratios) to $82-98 \mathrm{wt} \%$ (after pyrolysis at $550-800$ and $1000{ }^{\circ} \mathrm{C}$ ). Thus, it seems that as a result of the incorporation of the carbon black, the aerogels are more resistant to surface capillary pressures, thus allowing pyrolysis with nearly no structural shrinkage/collapse. This is also supported by large carbon yield of the gel/CCA composites, compared to the conventional synthesis, as mentioned above. The enhanced resistance of the gel primary particles to collapse upon annealing would be attributed to their large porosity and the reinforcement effect of the carbon black in the matrix, in a similar way as that reported for some resins or fibers-reinforced gels [35-37].

\subsection{Electrochemical characteristics in aqueous medium}

To investigate the potential applications of the synthesized carbon gels as electrodes in various electrochemical systems, we have measured the electrical resistivity of the carbon gels prepared with and without the CCA. As seen in Table 2, the resistivity values of the carbon gels prepared by the conventional sol-gel process showed a decreased trend with the R/C 
ratio; this has been explained in terms of the higher density of defects typically present in polymeric gels [38]. After incorporation of the carbon black additive, the resistivity decreased by an order of magnitude for all the synthesized materials, and the trend with the $\mathrm{R} / \mathrm{C}$ is still subtly observed (Fig. 5). The resistivity values are also lower (i.e. higher conductivity) than those reported for amorphous carbon materials (Fig. 5) typically used in electrochemical applications $[23,24]$. For a given $\mathrm{R} / \mathrm{C}$ molar ratio, similar resistivity values were obtained for the aerogels prepared with 5 and 10 wt.\% of the CCA (Table 2). Lower carbon black loadings did not seem to affect the electrical conductivity of the gels, whereas higher loadings were not achievable (lack of homogenous dispersion). All this points out that the resistivity of the prepared materials does not only depend on a critical carbon black loading, but also on the interconnectivity between the CCA particles and the RF clusters during the synthesis.

Table 2. Resistivity values of the synthesized carbon aerogels and gel/CCA nanocomposites.

\begin{tabular}{|c|c|c|c|c|}
\hline & $\begin{array}{l}\overline{\text { Resistivity }} \\
\text { [Ohm sq] }\end{array}$ & $\mathrm{R} / \mathrm{C}$ & $\begin{array}{c}\text { CCA } \\
\text { [wt. \%] }\end{array}$ & $\begin{array}{c}\text { Pyrolysis Temp } \\
{\left[{ }^{\circ} \mathrm{C}\right]}\end{array}$ \\
\hline Carbon Black (CCA) & 2.8 & n.a. & $100 \%$ & n.a. \\
\hline G25 & 278 & 25 & none & 800 \\
\hline G50 & 247 & 50 & none & 800 \\
\hline G100 & 223 & 100 & none & 800 \\
\hline G200 & 202 & 200 & none & 800 \\
\hline G25-CB10 & 63 & 25 & 10 & 800 \\
\hline G50-CB10 & 58 & 50 & 10 & 800 \\
\hline G100-CB10 & 25 & 100 & 10 & $1000(800)$ \\
\hline G200-CB10 & 22 & 200 & 10 & 1000 \\
\hline G50-CB5 & 53 & 50 & 5 & 800 \\
\hline
\end{tabular}




\begin{tabular}{lcccc} 
G50-CB10-T500 & $\infty$ & 50 & 10 & 500 \\
G50-CB10-T1000 & 29 & 50 & 10 & 1000 \\
\hline
\end{tabular}

An important parameter affecting the electrical features of the gels is the pyrolysis temperature; resistivity values of all as-prepared materials before carbonization (not shown) and those carbonized at $500{ }^{\circ} \mathrm{C}$ were extremely high, corresponding to non-electrically conductive materials. Although the low conductivity of RF carbon gels carbonized at temperatures below $600{ }^{\circ} \mathrm{C}$ has been reported for carbon gels prepared by the conventional method [39], it was rather surprising to obtain such high resistivity values in the materials prepared by incorporating ca. 10 or $5 \mathrm{wt} . \%$ of an electrically conductive additive (even for those treated at $500{ }^{\circ} \mathrm{C}$ ). Increasing the pyrolysis temperature above $500{ }^{\circ} \mathrm{C}$ decreased the resistivity values, with similar values for the gels treated at 800 and $1000{ }^{\circ} \mathrm{C}$.

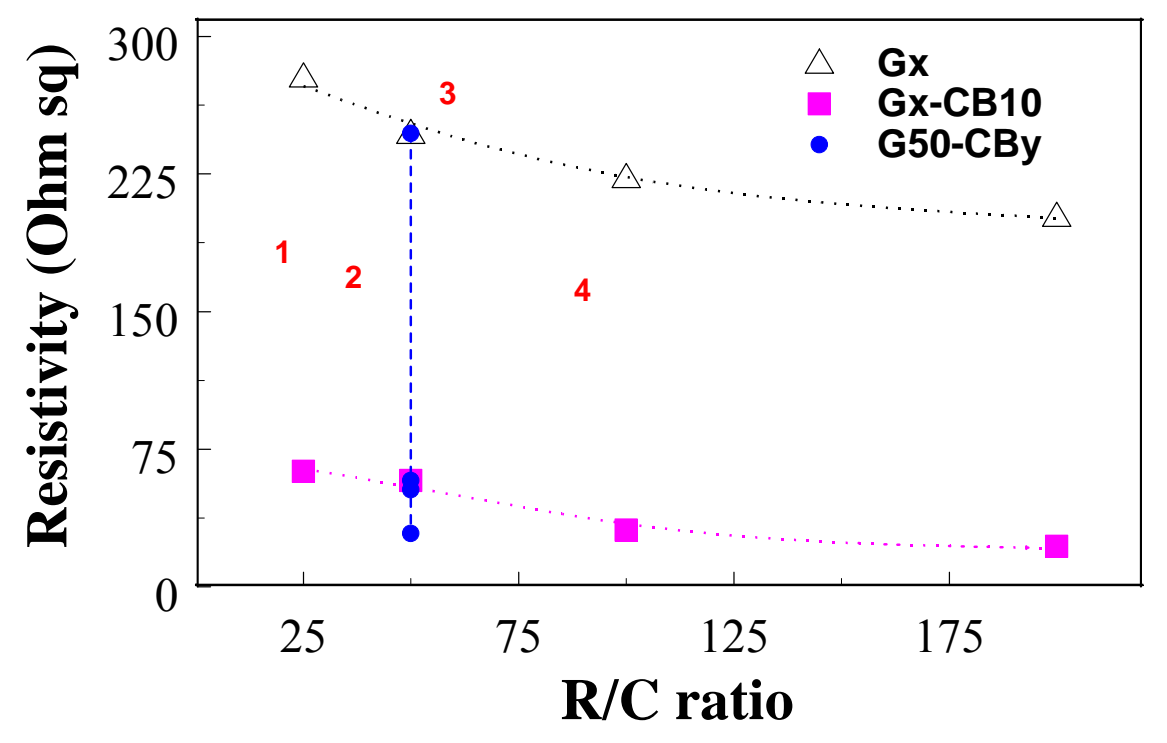

Fig. 5. Correlation between the electrical resistivity and the $\mathrm{R} / \mathrm{C}$ ratio of the series with (squares) and without CCA (triangles). Circles account for the resistivity of sample G50-CBy with increasing amounts of carbon black (ca. $y=0,5$ and 10wt.\%); lines 
are guides for the eyes. Resistivity values of reference carbons are also included for comparison: (1) Norit activated carbon; (2) Maxsorb activated carbon; (3) coconut shell-activated carbon; (4) bituminous coal activated carbon.

These observations were corroborated by cyclic voltammetry of the materials in an aqueous electrolyte shown in Fig. 6, where the poor performance after pyrolysis at $500{ }^{\circ} \mathrm{C}$ contrasts with the response upon treatment 800 and $1000{ }^{\circ} \mathrm{C}$ for a given sample (i.e., G50-CB10). Thus electrical resistivity of the gel/CCA composites arises from the surface moieties decorating the carbonaceous matrix of the gel (mainly ether bridges and $\mathrm{OH}$ groups) which are not removed unless high temperatures are applied. Consequently, the electrical conductivity of the gel/CCA composites is greatly defined by the chemical composition of the carbon matrix and to a lesser extent by its texture.

The large pore volumes and low electrical resistivity of the synthesized gel/CCA nanocomposites anticipate a good performance as electrodes, further corroborated by the electrochemical response of the obtained materials analyzed in a three electrode cell configuration in neutral aqueous electrolyte. Cyclic voltammograms of selected materials (representative of the series) are shown in Fig. 6, for a potential cut-off hold between -800 and $+800 \mathrm{mV}$ vs NHE. Despite the thermodynamic potential for water decomposition (i.e. -380 $\mathrm{mV}$ vs NHE) was largely surpassed, no current leaps related with gas evolution due to the decomposition of the electrolyte were observed. This behavior is rather common for porous carbon electrodes in sodium sulfate electrolyte [40]; it has been attributed to the high overpotential for $\mathrm{H}_{2}$ evolution of neutral electrolyte, and the ability of porous carbon electrodes to store the nascent hydrogen below $-400 \mathrm{mV}$ vs NHE. The wide humps observed in the anodic sweeps between $+200-400 \mathrm{mV}$ vs NHE (see for instance G200-CB10) confirmed the electrooxidation of the hydrogen stored on the electrodes. 
Other than the humps due to electrooxidation/reduction of nascent hydrogen, the voltammograms showed the ideal rectangular shape associated to the charge/discharge of the electric double layer (pure capacitive behavior). Current peaks arising from pseudo-faradaic reactions involving redox charge transfer reactions associated to surface groups were not detected, which is in agreement with the low oxygen content of the pyrolyzed aerogels. As mentioned above, the gels treated at $500{ }^{\circ} \mathrm{C}$ showed a negligible electrochemical response, in accordance with the resistivity values measured for the electrodes (Table 2). This contrasts with the behavior widely reported for porous carbon electrodes where the incorporation of oxygen functionalities often leads to enhanced capacitance values due to the pseudocapacitance contribution linked to surface functionalities [41]. Having a rich surface chemistry, it appears that the oxygen moieties decorating the carbon matrix of the gels pyrolyzed at $500{ }^{\circ} \mathrm{C}$ are not electrochemically active. Thus, pyrolysis temperature is critical to assure the good electrochemical performance of carbon gels, even if a conductive additive is incorporated in the synthesis. 

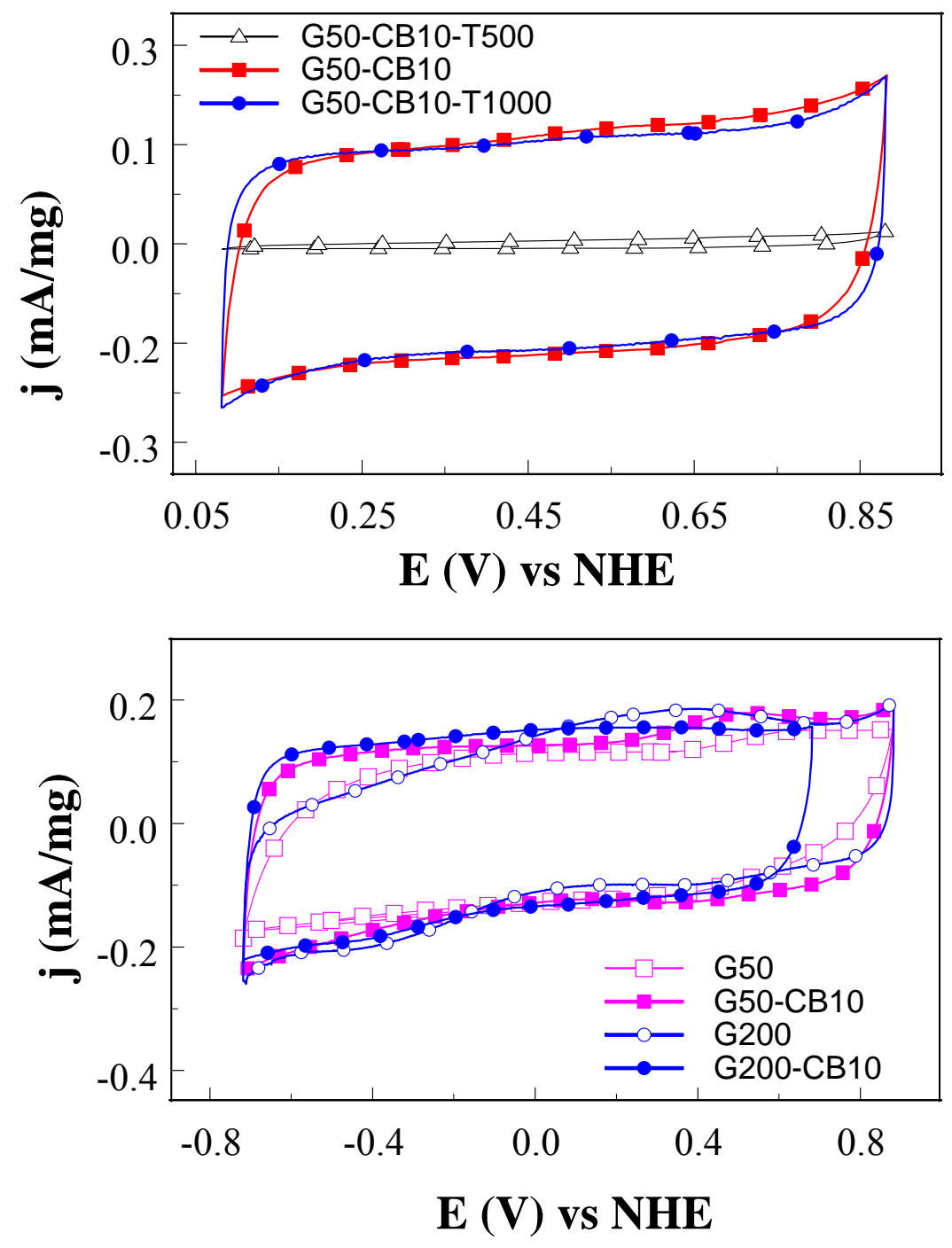

Fig. 6. (A) Effect of the pyrolysis temperature on the electrochemical response of the carbon gels modified with the CCA during the synthesis. (B) Cyclic voltammograms in $0.5 \mathrm{M} \mathrm{Na}_{2} \mathrm{SO}_{4}$ of selected carbon aerogels prepared with and without the CCA.

The electrodes pyrolyzed at 800 and $1000{ }^{\circ} \mathrm{C}$ also showed a stable electrochemical response, being reversibly charged/discharged at large potential windows (ca. 1.4 V) in sodium sulfate and over several hours, without significant losses in the current density nor the appearance of new waves in the voltammograms that would be arising from the oxidation of the carbon 
matrix. Such large potential windows in a neutral electrolyte have been reported for some carbon electrodes and attributed to the high overpotential for hydrogen evolution and their resiliency to anodic oxidation [40,42], although this is not a common characteristic of most carbon materials (which become easily oxidized under anodic conditions) [43]. Fig. 7 shows the evolution of the specific cell capacitance of selected electrodes upon aging at a constant cell voltage of $1.4 \mathrm{~V}$. The results showed a pattern related to the $\mathrm{R} / \mathrm{C}$ ratio, which become more pronounced for the gel/CCA composites. The extent of the capacitance fading decreased for the materials with high $\mathrm{R} / \mathrm{C}$ ratio, and that the electrodes of the series $\mathrm{Gx}$ faded faster than those of the gel/CCA materials. The cycle life of these electrodes is improved, likely as a result of the resiliency introduced by the carbon black within the aerogels framework.

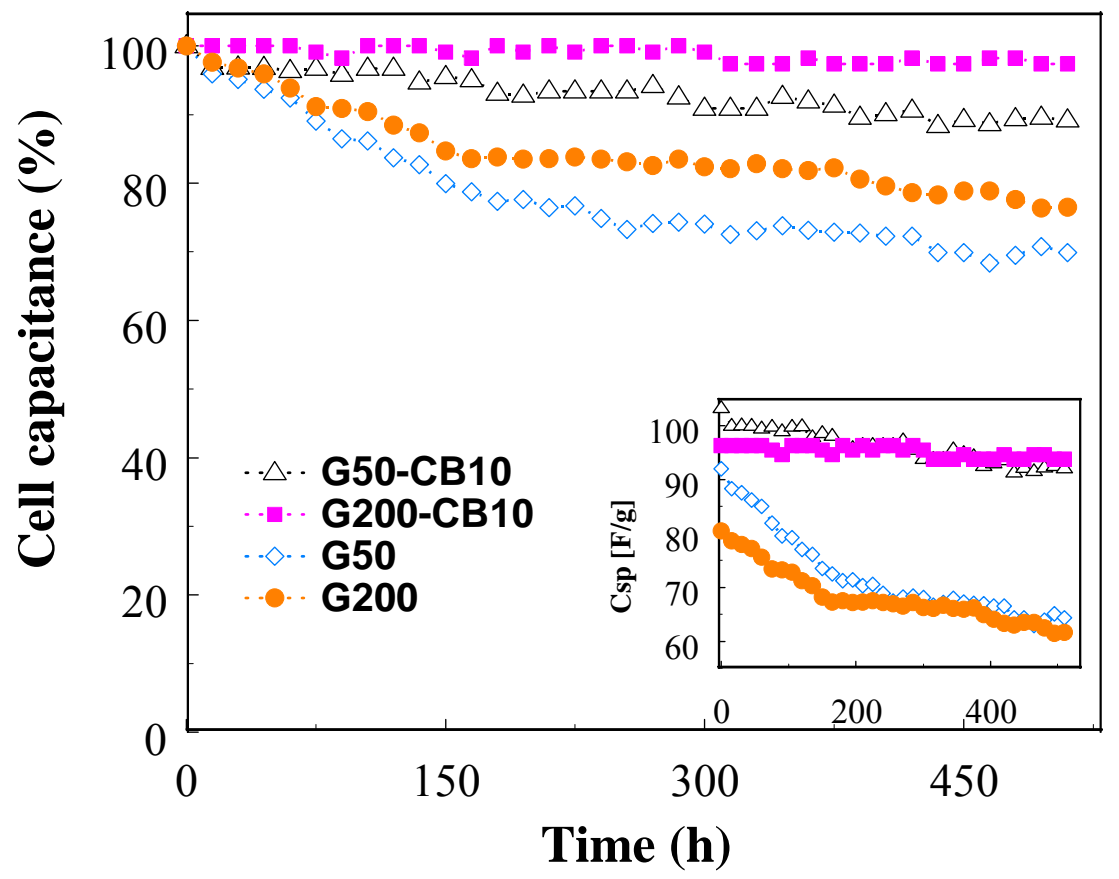

Fig. 7. Loss of specific cell capacitance -symmetric aerogel-based capacitor- during aging at constant voltage load tests at $1.4 \mathrm{~V}$ in $0.5 \mathrm{M} \mathrm{Na}_{2} \mathrm{SO}_{4}$. Inset shows the specific capacitance values obtained for the studied aerogel samples. 
As a drawback, all the prepared gel/CCA materials showed limited capacitance values in aqueous medium (ca. 110-120 and 110-85 F/g in $\mathrm{H}_{2} \mathrm{SO}_{4}$ and $\mathrm{Na}_{2} \mathrm{SO}_{4}$, respectively, both measured at $200 \mathrm{~mA} / \mathrm{g}$ ), close to the values of the gels prepared following the conventional synthesis (series Gx) but smaller than those reported for doped carbon electrodes [8, 14-16]. This is attributed to the similar microporosity of all gel/CCA samples with values around $0.20 \mathrm{~cm}^{3} \mathrm{~g}^{-1}$, and in agreement with those usually reported for carbon aerogels $[5,6,21,22]$. Although the capacitance values of these aerogels could be significantly improved by increasing their microporosity by physical or chemical activation without losses in the mechanical strength, the combination of large mesoporosity and excellent electrochemical performance make these materials ideal candidates to be used as conductive porous supports for the immobilization of large molecules (i.e., enzymes) of electrochemical interest. Moreover, the possibility to operate at a large electrochemical window coupled to the enhanced life cycles of these gel/CCA nanomaterials offers an interesting strategy for those applications where a high potential /voltage and good reversibility are desired (for instance capacitive deionization, electrochemical, energy storage, or biosensors). Additionally, the high resistance to electro-oxidation of the prepared carbon gels overcomes the limitation of most porous carbons in electrochemical applications (i.e. activated carbons), becoming an interesting feature to be further explored. Future research will focus on adjusting the micro/mesopore ratio and pore volumes in the low-nanometer scale, since this would be expected to expand the applicability of the materials.

\section{CONCLUSIONS}

We have synthesized electrically conductive and highly mesoporous carbon gels by a simple modification of the traditional resorcinol-formaldehyde catalyzed polycondensation route 
widely described in the literature for the preparation of carbon gels. The polycondensation of the reactants in the presence of a carbon conductive additive allowed a facile preparation of electrically conductive carbon aerogels with a unique combination of ultrahigh mesopore volumes and low electrical resistivity. The conductive additive had a large impact on the catalyzed sol-gel polymerization of the reactants, directing the synthesis towards the formation of less-branched clusters and of polymer particles with large sizes; this allowed a fine-tuning control over the micro/mesoporosity of the gel/CCA materials, while providing electrical conductivity within the polymer carbon gel particles.

Connectivity of the large mesopore cavities by large narrow necks assured an efficient charge/discharge rate in electrochemical applications due to the enhanced diffusion of ions in the large connected channels. The materials pyrolyzed above $500{ }^{\circ} \mathrm{C}$ showed stable electrochemical response in neutral aqueous electrolyte, being reversibly charged/discharged at large potential windows over several hundreds of cycles, without significant losses in the current density. Another important feature related to the incorporation of the carbon black during the sol-gel step is the better life cycle of the resulting electrodes when charged/discharged at high voltage. These are interesting features for applications of carbon electrodes were a fast response is crucial.

\section{ACKNOWLEDGMENTS}

The authors thank the financial support of the Spanish MINECO and MMA (grants CTM2011/02338 and 083/RN08/03.3). Superior Graphite Co. is also acknowledged for kindly providing the carbon black. COA thanks Dr. Arenillas for kindly providing the resistivity measurements. MH thanks CSIC for her JAE-Doc contract.

\section{REFERENCES}


[1] Gogotsi Y, (Ed.) in Carbon Nanomaterials, CRC Press, Boca Raton, Florida, 2006.

[2] Tascón JMD (Ed.) in Novel Carbon Adsorbents, Elsevier Ltd., Amsterdam, 2012.

[3] Lee J, Kim T, Hyeon J. Recent progress in the synthesis of porous carbon materials. Adv Mater 2006;18:2073-94.

[4] Al-Muhtaseb SA, Ritter JA. Preparation and properties of resorcinol- formaldehyde organic and carbon gels. Adv Mater 2003;15:101-14.

[5] Job N, Thery A, Pirard R, Marien R, Kocon L, Rouzaud JN, et al. Carbon aerogels, cryogels and xerogels: Influence of the drying method on the textural properties of porous carbon materials. Carbon 2005;43:2481-94

[6] Job N, Pirard R, Marien R, Pirard JP. Porous carbon xerogels with texture tailored by $\mathrm{pH}$ control during sol-gel process. Carbon 2004;42:619-28.

[7] Tamon H, Ishizaka H, Araki T, Okazaki M. Control of mesoporous structure of organic and carbon aerogel. Carbon 1998; 36:1257-62.

[8] Calvo EG, Ania CO, Zubizarreta L, Menendez JA, Arenillas A. new routes in the synthesis of carbon xerogels for their application in electric double layer capacitors. Energy \& Fuels 2010;24:3334-9.

[9] Zubizarreta L, Arenillas A, Pirard JP, Pis JJ, Job N, Tailoring the textural properties of activated carbon xerogels by chemical activation with $\mathrm{KOH}$. Microp Mesop Mater 2008;115:480-90.

[10] Pekala RW. Organic aerogels from the polycondensation of resorcinol with formaldehyde. J Mater Sci 1989;24(9):3221-7.

[11] Yamamoto T, Endo A, Ohmori T, Nakaiwa M. Porous properties of carbon gel microspheres as adsorbents for gas separation. Carbon 2004;42:1671-6.

[12] Moreno-Castilla C, Maldonado-Hodar FJ. Carbon aerogels for catalysis applications: An overview. Carbon 2005;43:455-65. 
[13] Pajonk GM. Aerogel Catalysts. Appl. Catal. 1991;72:217-66.

[14] Pekala RW, Farmer JC, Alviso CT, Tran TD, Mayer ST, Miller JM, et al. Carbon aerogels for electrochemical applications. J. Non-Cryst. Solids 1998;225:74-80.

[15] Li W, Reichenauer G, Fricke J. Carbon aerogels derived from cresol-resorcinolformaldehyde for supercapacitors. Carbon 2002;40:2955-59.

[16] Gutiérrez MC, Picó F, Rubio R, Amarilla JM, Palomares FJ, Ferrer ML, et al. PPO15PEO22-PPO15 block copolymer assisted synthesis of monolithic macro- and microporous carbon aerogels exhibiting high conductivity and remarkable capacitance. J Mater Chem 2009;19:1236-40.

[17] Lee YJ, Jung JC, Park S, Seo JG, Baeck SH, Yoon JR, et al. Preparation and characterization of metal-doped carbon aerogel for supercapacitor. Curr Appl Phys 2010;10:947-51.

[18] Farmer JC, Fix DV, Mack GV, Pekala RW, Poco JF. Capacitive deionization of $\mathrm{NH}_{4} \mathrm{CLO}_{4}$ solution with carbon aerogel electrodes. J Appl Electrochem 1996;26:100718.

[19] Haro M, Rasines G, Macias C, Ania CO. Stability of a carbon gel electrode when used for the electro-assisted removal of ions from brackish water. Carbon 2011;49:3723-30.

[20] Xu P, Drewes JE, Heil D, Wang G. Treatment of brackish produced water using carbon aerogel-based capacitive deionization technology. Water Res 2008;42:2605-17.

[21] Fellinger TP, White RJ, Titirici MM, Antonietti M. Borax-Mediated Formation of Carbon Aerogels from Glucose. Adv Funct Mater 2012;22:3254-60.

[22] Szczurek A, Amaral-Labat G, Fierro V, Pizzi A, Masson E, Celzard A. The use of tannin to prepare carbon gels. Part I: Carbon aerogels. Carbon 2011;49:2773-84.

[23] Pandolfo AG, Hollenkamp AF. Carbon properties and their role in supercapacitors. J Power Sources 2006;157:11-27. 
[24] Park K, Lee J, Park P, Yoon S, Moon J, Eum H, et al. Development of a carbon sheet electrode for electrosorption desalination. Desalination 2007;206:86-91.

[25] Nadakatti S, Tendulkar M, Kadam M. Use of mesoporous conductive carbon black to enhance performance of activated carbon electrodes in capacitive deionization technology. Desalination 2011;268:182-8.

[26] Xi-miao L, Rui Z, Liang Z, Dong-hui L, Wen-ming Q, Jun-he Y, et al. Impedance of carbon aerogel/activated carbon composites as electrodes of electrochemical capacitors in aprotic electrolyte. New Carbon Materials, 2007;22:153-8.

[27] Rasines G, Lavela P, Macias C, Haro M, Ania CO, Tirado JL. Electrochemical response of carbon aerogel electrodes in saline water. J Electroanal Chem 2012;671:92-8.

[28] van der Pauw J. A Method of Measuring Specific Resistivity and Hall Effect of Discs of Arbitrary Shapes. Philips Res Repts, 1958;13:1-9.

[29] Renou J, François-Rossetti J, Imerick B. Etudes des solides poreux. Isotherms d'adsorption irregulieres. Bul. Soc. Chimique de France, 1961;91:446-50.

[30] de Boer JH. in The Structure and Properties of Porous Materials, (Eds. D. H. Everett and F. S. Stone), Butterworths, 1958 pp. 84-145.

[31] Van Der Voort P, Ravikovitch PI, De Jong KP, Neimark AV, Janssen AH, Benjelloun M, et al. Plugged hexagonal templated silica: a unique micro- and mesoporous composite material with internal silica nanocapsules, Chem Comm 2002;9:1010-1.

[32] Phuong T, Nguyen M, Do DD, Nicholson D. On The Cavitation and Pore Blocking in Cylindrical Pores with Simple Connectivity. J Phys Chem B 2011;115:12160-72.

[33] Vishnyakov A, Neimark AV. Monte Carlo Simulation Test of Pore Blocking Effects. Langmuir 2003;19:3240-7. 
[34] Rasmussen CJ, Vishnyakov A, Thommes M, Smarsly BM, Kleitz F, Neimark AV. Cavitation in Metastable Liquid Nitrogen Confined to Nanoscale Pores. Langmuir 2010;26:10147-57.

[35] Petricevic R, Glora M, Fricke J. Planar fibre reinforced carbon aerogels for application in PEM fuel cells. Carbon 2001;39:857-67.

[36] Saliger R, Fischer U, Herta C, Fricke J. High surface area carbon aerogels for supercapacitors. J. Non- Cryst Solids 1998;225:81-5.

[37] Petricevic R, Reichenauer G, Bock V, Emmerling A, Fricke J. Structure of carbon aerogels near the gelation limit of the resorcinol-formaldehyde precursor. J Non- Cryst Solids 1998;225:41-5.

[38] Wang J, Zhang SQ, Shen J, Guo YZ, Attia SM, Zhou B, et al. Electrical transport properties of carbon aerogels. J Porous Mater 2001;8:167-70.

[39] Lin C, Ritter JA, Popov BN. Correlation of Double-Layer Capacitance with the Pore Structure of Sol-Gel Derived Carbon Xerogels. J Electrochem Soc 1999;146,:3639-43.

[40] Demarconnay L, Raymundo-Piñero E, Beguin F. A symmetric carbon/carbon supercapacitor operating at $1.6 \mathrm{~V}$ by using a neutral aqueous solution. Electrochem Comm 2010;12:1275-8.

[41] Raymundo-Piñero E, Beguin F. in Activated Carbon Surfaces in Environmental Remediation, vol 7 (T. J. Bandosz, Ed), Elsevier, Oxford, 2006, chapter 6.

[42] Bichat MP, Raymundo-Piñero E, Béguin F. High voltage supercapacitor built with seewead carbons in neutral aqueous electrolyte, Carbon 2010;48:4351-4361.

[43] Berenguer R, Marco-Lozar JP, Quijada C, Cazorla-Amoros D, Morallon E. Effect of electrochemical treatments on the surface chemistry, Carbon 2009;47:1018-1027. 


\title{
Supplementary Information
}

Carbon black directed synthesis of ultrahigh mesoporous carbon aerogels Carlos Macías ${ }^{1}$, Marta Haro ${ }^{2}$, José B. Parra ${ }^{2}$, Gloria Rasines ${ }^{1}$, Conchi O. Ania ${ }^{2}$ *

\author{
${ }^{1}$ Nanoquimia S.L., P.L La Minilla, La Rambla, 14540 Córdoba (Spain) \\ ${ }^{2}$ Instituto Nacional del Carbón, INCAR-CSIC, Apdo. 73, 33080 Oviedo, Spain
}

*Corresponding author. Tel/Fax: +34 985 118846/297662.

E-mail address: conchi.ania@incar.csic.es (C.O. Ania) 


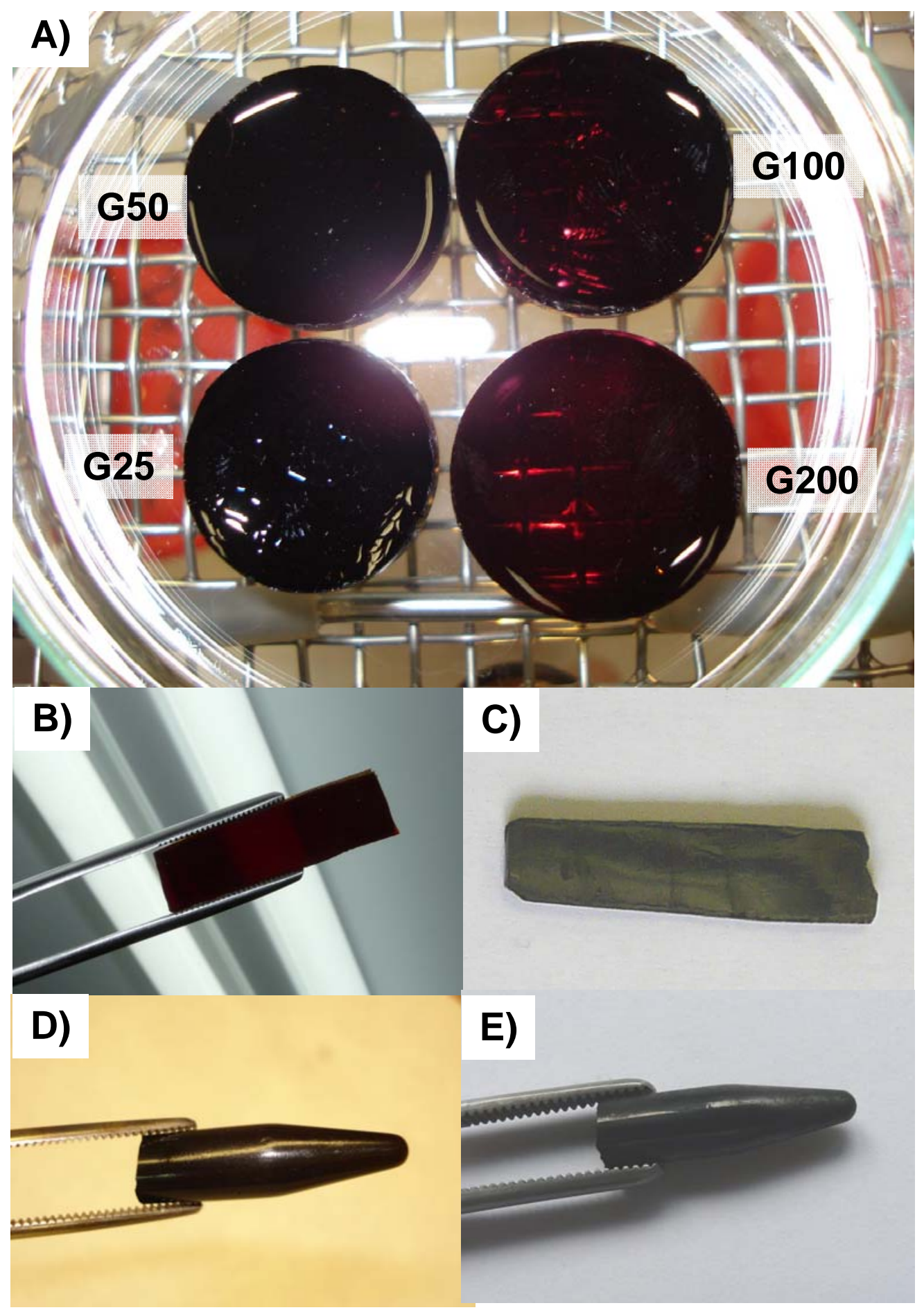

Fig. S1 Images of the prepared carbon aerogels with and without the addition of the conductive additive. (A) gels from the conventional synthesis before pyrolysis; (B) sample G200 before pyrolysis; (C) sample G200 after pyrolysis; (D) G200-CB10 before pyrolysis and (E) G200-CB10 after pyrolysis. 